ISSN: $1130-3743$

\title{
LA ETNOGRAFÍA EN LA FORMACIÓN DE ENSEÑANTES
}

\section{The Ethnography and the training of teachers}

\author{
María BERTELY BUSQUETS \\ Centro de Investigaciones y Estudios Superiores en Antropología Social (CIESAS). \\ Juárez 87, Tlalpan 14000. México,D.F. bertely@juarez.ciesas.edu.mx
}

Fecha de aceptación definitiva: marzo de 2002

BIBLID [(1130-3743) 13, 2001, 137-160]

\section{RESUMEN}

Este artículo responde a la pregunta ¿cómo podemos formar mejores maestros en nuestras escuelas y para nuestras escuelas? La autora propone que la investigación etnográfica en educación puede articularse a la formación docente a partir de varias rutas. Una vía de articulación puede derivarse de la implicación subjetiva de los profesores en las preguntas que se plantean en torno a la cultura educacional. Otro sendero se relaciona con la habilidad de los maestros para adquirir competencias etnográficas específicas. El tercero se refiere a la construcción de "teorías educativas arraigadas". Finalmente, la propuesta de combinar la investigación etnográfica con la formación de maestros depende de la capacidad de organización de los actores involucrados. Esto significa que los maestros, investigadores, líderes comunitarios, diseñadores de políticas y otros, deberían dialogar acerca de sus perspectivas en torno a las prácticas educativas.

Palabras clave: Etnografía, formación docente, implicación subjetiva, competencias etnográficas, epistemología, teoría educativa arraigada

\section{SUMMARY}

This paper answers the question: how can we train better teachers in our schools and for our schools? The author proposes than the ethnographic educational research can be articulated to the training of teachers by several rutes. One way of articulation can be the subjective implication of the teachers themselves in the questions related to the educational culture. Another way relays on the ability of 
the teachers to adquire specific ethnographic competences. A third would be the construction of "grounded educational theories". Finally the whole proposal to combine the ethnographic research with the training of teachers depends on the capacity of organization of the involved actors. It means that teachers, researchers, communitary leaders, educational politicians and others, should speak about their perspectives on educational practices.

Key words: Ethnography, training teachers, subjetive implication, ethnographic competences, epistemology, grounded educational theory.

¿Cómo formar mejores enseñantes en y para las escuelas? Algunos etnógrafos educativos que iniciamos nuestra carrera en la docencia (Bertely en Rodríguez (coord.), 1999; Bertely, 2000; López Miranda, 2000), intentamos responder a esta pregunta estableciendo algunas identificaciones entre nuestra propia trayectoria académica y los senderos que tendrían que recorrer los profesores en proceso de formación. A partir de mi propia trayectoria, me inclino por una profesionalización docente anclada en lo que llamo el preguntar etnográfico y en la posibilidad de relacionar las perspectivas cualitativas aplicadas a la investigación educativa con los procesos de formación.

El primer sendero, al que denomino de la implicación, supone descubrir el horizonte subjetivo que antecede a las preguntas de los enseñantes, propiciar las quiebras y los procesos de autorreflexión relacionados con las sorpresas derivadas, así como estimular el uso de nuevos géneros narrativos en las etnografías escolares y áulicas producidas. El segundo sendero, al que defino formativo, implica determinar el lugar que ocupa el dominio de determinadas competencias etnográficas en la formación de mejores maestros y en la búsqueda de las respuestas a sus preguntas de investigación.

Por su parte, el sendero interpretativo alude a la necesidad de propiciar la generación de teorías educativas arraigadas, definidas como las formalizaciones analíticas y conceptuales que, ancladas en la capacidad estratégica, las experiencias cotidianas y las preguntas de los mismos docentes, se traduzcan en una efectiva transformación de las representaciones y las prácticas educativas, así como en la configuración de nuevos imaginarios que vitalicen identidades y comunidades escolares en crisis. Por último, el sendero democrático, alude al significado que adquiere para el etnógrafo educativo la inclusión de nuevas realidades, voces y actores, así como el diálogo, en la construcción de comunidades de indagación incluyentes y plurales. 


\section{El SENDERO DE LA IMPLICACIÓN SUBJETIVA: AUTORREFLEXIÓN, QUIEBRAS Y NUEVOS GÉNEROS ETNOGRÁFICOS}

La riqueza informativa que descansa en las trayectorias de vida y escolares de los profesores, así como el proceso de autorreflexión que se deriva de su trabajo etnográfico en las comunidades, las escuelas y los salones de clases, plantean como necesario el uso de nuevos géneros narrativos en sus reportes o, en otras palabras, de escrituras etnográficas que muestren el valor formativo de la implicación del actor en la realidad que estudia.

\subsection{Trayectorias personales, implicación subjetiva y autorreflexividad}

En el Coloquio Internacional Educación, Humanismo y Posmodernidad, realizado en México a iniciativa de la Universidad Pedagógica Nacional en el año 2000, tuve la oportunidad de replicar la conferencia La Gente Buena Existe de Judith Friedlander, además de participar en el debate que se dio, en ese mismo evento, en torno a los aportes de Agnes Heller. Uno de los núcleos centrales del debate consistió en analizar la necesidad de reintegrar el sentido humanista a la sociedad posmoderna, lo cual me remitió al problema de la implicación del sujeto en la historia, planteado por Heller en su obra la Teoría de los Sentimientos (1993). La autora sostiene en esta obra que "sentir es estar implicado en algo", un concepto, una persona, uno mismo, un proceso, un problema, otro sentimiento. Me encuentro implicado, afirma, si lo que siento se relaciona conmigo, con mis ideas, con mis objetivos y con las circunstancias de mi vida. En este marco interpretativo, coincido también con Friedlander en que la gente buena "y en consecuencia el buen profesor" debería ser aquella que se encuentra implicada en la cotidianeidad de su tiempo histórico.

Hablar de un docente implicado alude a la subjetividad que antecede al preguntar etnográfico y a la configuración ética de un sujeto histórico situado aquí y ahora, además de conducirnos al reconocimiento de las múltiples verdades, culturas, formas de vida y filosofías, ancladas en una aspiración de libertad universal distinta a la apuntalada en la verdad positiva. Heller y Friedlander hablaron entonces de intelectuales que, desde el campo de la filosofía, la política, la sociología, la antropología y el arte, se identifican entre sí y con otros seres humanos "no importando las fronteras geográficas", por el solo hecho de estar implicados en algo y compartir preocupaciones éticas y sentimientos similares.

Al hombre ético de hoy, en tanto implicado, le duelen los horrores, los padecimientos y las exclusiones asociados con la vigencia de los fundamentalismos porque, en muchos casos, ha padecido los mismos horrores y exclusiones en él y en su experiencia con los otros. El hombre ético de hoy reconoce su humanidad y no se aparta de ella para analizarla objetivamente; el hombre ético de hoy se asume como sujeto en el mundo, sujeto histórico, sujeto cultural prototípico, que 
se pregunta desde una trayectoria y experiencia de vida, anticipando el sentido de las respuestas.

Cuando Friedlander, en la ponencia citada, describe al oficial italiano que en la Primera Guerra Mundial se negó a matar a su enemigo austríaco porque reconoció en su rango militar, en su uniforme, en el blanco de sus ojos, en sus movimientos, en su habla y en sus formas de comer y saborear el café algo extrañamente familiar, recuerdo el modo en que los informantes que han colaborado en mis investigaciones me han parecido, en sus preocupaciones, en sus satisfacciones y en sus expectativas escolares, extrañamente familiares. Familiaridad que me implica necesariamente en los sufrimientos, las paradojas y las disyuntivas de nuestro tiempo, asociadas "en mi caso" con las prácticas educativas de inclusión y exclusión que configuran mi identidad y las de otros. Al estar implicado y dispuesto a implicarme en otros horizontes significativos, he podido descubrirme como particular y distinto al otro, un otro distinto a mí y, a la vez, tan humano como yo.

En los últimos años me he percatado del significado que tiene la implicación o interioridad subjetiva en la configuración de las preguntas etnográficas que plantean los profesores en formación, usualmente justificadas a partir de los referentes políticos, estadísticos y teóricos que les permiten validar la relevancia social e histórica de sus objetos de estudio (Galindo, 1993).

Con el objeto de mostrar la validez empírica de este argumento y construir estrategias formativas adecuadas que estimulen en los profesores el deseo de interpretar la cultura escolar y lo que sucede en los salones de clases, he solicitado a mis alumnos "aproximadamente sesenta profesores que cursan programas de Maestría y Doctorado", escribir en torno a sus trayectorias personales. He comprobado, en todos los casos, cómo sus preguntas etnográficas se relacionan con preocupaciones que les son vitales, que los remiten a experiencias de vida y escolares tempranas vinculadas con su configuración identitaria y donde, en consecuencia, la discriminación de lo uno y de lo otro, en sus narrativas escritas acerca de lo vivido en y más allá de las escuelas, está presente. En todos los casos se identifican marcas que podrían calificarse como francas obsesiones, a partir de las cuales se explica la diversidad de preguntas y, en el caso de los etnógrafos educativos consolidados, la fascinación que mostramos por determinados temas.

El reconocimiento de la implicación subjetiva no supone, sin embargo, la existencia de individuos aislados que se preguntan y responden "como locos en el mundo" acerca de realidades que nada comprenden otros, sino de sujetos sociales que, en tanto sujetados a entramados históricos y culturales específicos y como actores prototípicos, comparten experiencias de vida y escolares, códigos de interacción y preocupaciones que resultan para amplios sectores sociales, no sólo extrañamente familiares, sino profundamente significativas.

A continuación, incluyo un fragmento de la trayectoria escolar narrada por una profesora mexicana, narración que muestra el anclaje subjetivo de la pregunta etnográfica que plantea, así como las razones que explican su interés por interpretar las 
repercusiones de la privación del recreo escolar en los alumnos que asisten a una escuela primaria ${ }^{1}$ :

Vivir en una ciudad como Nezahualcóyotl, estado de México, hace veintiocho años, resultaba verdaderamente difícil. No obstante se le denominaba "ciudad" carecía de agua potable, drenaje, banquetas, pavimento y luz. Aunque esta ciudad se empezaba a formar ya contaba con escuelas primarias. Aún recuerdo con cierta melancolía mi primera escuela, donde estudié la Primaria. Era una escuela con dieciséis grupos y con un patio de recreo muy pequeño, donde era difícil correr, aunque no imposible. En tiempo de lluvias era impresionante y divertido ver cómo esquivábamos los charcos y montones de tierra para llegar a nuestra escuela. La mayoría de mis compañeros éramos igual de pobres. La gente decía que Nezahualcóyotl se estaba formando con gente que había huido de sus pueblos y que apenas tenía dinero para construir una casa de lámina.

Maestros que venían de la capital de la república o de algún lugar fuera de Nezahualcóyotl hacían una serie de comentarios al estilo «pobres niños!" y "ino sé cómo exigirles el uniforme si se ve que apenas tienen para comer!n. En esa escuela no se exigía el uniforme, ni los zapatos limpios, e inclusive se iba despeinado o sucio; el maestro hacía caso omiso del aseo. Pero algo que nunca pasó desapercibido era la disciplina, la autoridad y el poder que ejercía el maestro en el grupo. Nadie, ni los padres, protestaban. Al contrario, tal parecía que era parte de la educación que los maestros debían proporcionar y que los padres esperaban.

Recuerdo cómo el maestro Tomás, un hombre de carácter fuerte y muy exigente, negociaba con Norberto y José la salida al recreo. Ellos decían que por favor los dejara salir porque "les tocaba" jugar con los niños de tercero. El maestro les puso un reto: "si aguantan cinco golpes de palo atrás de las rodillas, con las manos extendidas y agarrándose del marco de la puerta del salón, pueden salir". El maestro sugería con ello que sólo de esa manera podían "pagar" todo el desorden que habían ocasionado en el salón durante el día.

Norberto y José, en su afán de salir al recreo, se dejaron golpear mientras los demás nos quedamos paralizados observando cómo el maestro descargaba toda su fuerza contra las piernas de nuestros compañeros de clase. Asumido el castigo, ellos se habían ganado la salida al recreo aunque, por los golpes que les dieron y el dolor, no pudieron jugar fútbol como lo habían planeado. El ardor de piernas no se los permitió. A todos nos sirvió de experiencia lo sucedido y aprendimos a no negociar con el maestro la salida al recreo.

Aún siento aquella tristeza, angustia, desesperación e impotencia ante el maestro cuando señalaba con su dedo y decía: "jtú, no sales porque no trajiste la tarea!", "tú, no sales porque estuviste jugando!", "tú no, porque te paraste!" y "jtú no, porque te la pasaste volteando y no atendiste a la clase! . A mí me impresionaba la forma tan rápida que tenía el maestro para decidir, en menos de cinco minutos, quién salía y quién no salía al recreo. Cuando dejaba a mis amigas sin recreo se derrumbaban

1. El contexto de la narración es Ciudad Nezahualcóyotl, una colonia urbana popular de las orillas de la capital de la República Mexicana, en la frontera entre la zona metropolitana y el estado de México. 
todos los planes que habíamos construido en clase. El maestro no se daba cuenta de que en el recreo uno siempre se junta con los amigos predilectos y que sin ellos el recreo no resulta divertido. Al no salir el compañero con el que uno comparte esa media hora, tan apreciada, uno también se priva del recreo, pues en él uno comparte alimentos, alegrías, pleitos, juegos y pláticas.

Era tanto el anhelo de salir a jugar al patio de recreo, que la carita de tristeza y el rostro de hambre que mostraban mis compañeros a través de la ventana resultaba dramática. Lo peor no era eso, sino cuando el maestro Tomás se sentaba a comer con la maestra Blanca delante de los castigados esa exquisita comida que con el sólo olor despertaba más hambre de la que teníamos.

Realmente resultaba cruel permanecer en un salón de clases sin comer, haciendo sumas y copias larguísimas que eran impuestas por el maestro, con el propósito de que no las termináramos en la media hora que duraba el recreo. El ingenio de los que salíamos era más sorprendente aún, pues veíamos el modo de pasarles comida por debajo de la puerta y los huecos de los vidrios rotos a nuestros compañeros, sin que el maestro se diera cuenta. Cuando el maestro estaba de buenas les permitía comer la torta que traían; cuando no, los niños no probaban alimento en todo el día.

A la fecha, la falta de recreo como castigo sigue vigente en los salones de clases. Han pasado tantos años y aún sigo preguntándome: ¿por qué se priva al alumno del recreo? (Testimonio personal. Reina López González, alumna del Programa de Maestría del Instituto Superior de Ciencias de la Educación del Estado de México, Chalco, Estado de México, 1999).

En historias escolares como la narrada se encuentran las marcas de la implicación subjetiva que anteceden las preguntas etnográficas planteadas. Ahora comprendo por qué la pregunta de otra profesora, en torno a las causas de la deserción escolar en el tránsito entre la educación Primaria y la Secundaria, encontraba su anclaje subjetivo en que ella era la única de su familia que se había promovido en el sistema escolar hasta ingresar a un posgrado. Sus hermanos y hermanas, en contraste, habían desertado de la escuela, precisamente, después de concluir la escuela primaria.

Entiendo por qué un profesor que participó en grupos juveniles marginales, subalternos y contraculturales se preguntaba acerca del significado de la rebeldía y la actitud contracultural que manifestaban los alumnos en el nivel medio superior, en contraste con el proceso de normalización que experimentaban en las licenciaturas donde se formaban como docentes. Comprendo también por qué habiéndoseme prohibido interactuar con los niños que jugaban en la calle, yo misma me interesé en el estudio de los juegos que transgredian las reglas sociales y publiqué un artículo sobre los patrones de interacción infantil y el fútbol callejero.

Muchas son las trayectorias escolares y de vida pero, a final de cuentas, al plantear nuestras preguntas etnográficas con sinceridad "porque no todos los temas de investigación son genuinos y legítimos en sentido subjetivo", y al contestarlas desde un lugar, descubrimos que partimos de preguntas en las que estamos implicados y que nos son vitales. Resolver una pregunta etnográfica a partir del sendero 
de la implicación subjetiva lleva a los profesores en formación a descubrir, en pocas palabras, que sus interrogantes no se derivan de una realidad externa a sus propias experiencias culturales, escolares y de vida.

Otra demostración del impacto de la subjetividad en el intérprete se refiere también a las variaciones de los escritos etnográficos, planteada en el texto Antropologia Fenomenológica de Michael Agar (1982, en Reynoso (comp.), 1991). Dicha variedad, desde el enfoque hermenéutico, se deriva de:

- el entrenamiento profesional del etnógrafo donde, por ejemplo, el estudio de los métodos de crianza es documentado de distinta manera por freudianos, teóricos del aprendizaje y antropólogos;

- la historia personal del etnógrafo acerca de la cual George Devereux (1977), en su obra De la Ansiedad al Método en las Ciencias del Comportamiento, tiene mucho que aportar;

- el medio cultural del etnógrafo, a partir de lo cual se ha generado una crítica a la etnografía euroamericana a partir de indagaciones realizadas por los mismos nativos (Owusu, 1978);

- los cambios en el grupo descrito, como lo muestran las etnografías de Michael Agar en lo que denomina "la ecología química de las calles" (1977);

- la audiencia (colegas, etnógrafos, diseñadores de políticas, antropólogos y no antropólogos, académicos y no académicos); y

- la naturaleza siempre emergente del informe, analizada en la obra Grounded Theory (Glasser y Strauss, 1967).

El sendero de la implicación requiere que los futuros profesionales del preguntar etnográfico se apropien de las herramientas hermenéuticas que les permitan reconocer el lugar que ocupa la propia subjetividad en el proceso de interpretación.

La filosofía hermenéutica pone todas estas cuestiones bajo un solo paraguas. Ella afirma que la gente existe necesariamente dentro de una tradición, en términos de la cual se ve a sí misma, a su mundo, a su pasado y a su futuro. Un individuo nunca puede situarse por completo aparte y examinar su tradición como si fuese un objeto, porque fuera de ella no hay nada en términos de lo cual pueda tener lugar la comprensión. La comprensión, en otras palabras, tiene una "pre-estructura" (Gadamer, 1975) o una "dimensión tácita" (Polanyi, 1966) (Agar, 1982, 1991, 120).

\subsection{Implicación subjetiva y nuevos géneros etnográficos}

El sendero de la implicación subjetiva me remite de igual modo al Taller La Escritura Etnográfica ¿desde dónde y para quién? que se llevó a cabo en el IX Simposio Interamericano de Investigación Etnográfica en Educación, celebrado también en la Universidad Pedagógica de México en el año 2000. Nuestro objetivo consistía en mostrar los límites de las convenciones realistas que han caracterizado la producción 
de investigaciones etnográficas en educación, convencidos de que la subjetividad del intérprete irrumpe como iceberg en la antropología posmoderna.

Con este convencimiento, la lectura del artículo Las Etnografías como Textos (Marcus y Cushman, 1982, 1991) me llevó a reconsiderar el valor de las prácticas retóricas y narrativas asociadas al quehacer etnográfico, la autorreflexividad y la implicación subjetiva del docente en formación. Se trata de prácticas de escritura relacionadas con la experimentación etnográfica (Briggs, 1970; Rabinow, 1975; Rosaldo, 1980, 1980a), definida como:

[...] una reacción filosóficamente inspirada a las convenciones del género del realismo etnográfico, sobre el que ha existido un consenso tácito y artificial en la antropología anglonorteamericana durante aproximadamente los últimos 60 años. Es posible que esta experimentación no sólo esté alterando la naturaleza tradicional de la etnografía; puede más bien estar señalando el comienzo de una profunda reforma de las ambiciones teoréticas y de las prácticas de investigación [...]. La característica principal compartida por las etnografías experimentales es que integran, en sus interpretaciones, una preocupación epistemológica explícita en la forma en que se han construido tales interpretaciones y en las que se las presenta textualmente como discurso objetivo sobre los sujetos entre los cuales se ha conducido la investigación. [...] la escritura etnográfica contemporánea intenta sintetizar el debate clásico sobre la naturaleza hermenéutica (Palmer, 1969) [...] sobre la naturaleza de la interpretación [...] (Reynoso, 1991, 171-172).

Inspirada en estos autores me pregunté en qué medida solemos someter a los profesores que se forman a partir de la observación y la documentación de las prácticas escolares y docentes cotidianas, al conjunto de convenciones y pretensiones realistas inspiradas en la antropología norteamericana culturalista, en la tradición británica estructural y estructural-funcionalista, y en la ciencia social positiva. Una de las convenciones y pretensiones realistas más frecuente en la formación de etnógrafos educativos, incluidos los profesores, estriba en solicitar la ausencia de la primera persona en la producción de sus reportes de trabajo de campo, imaginando un narrador invisible, desapasionado, semejante a una cámara de grabación, donde el uso de la tercera persona otorga una supuesta autoridad científica. La perspectiva realista, sin embargo, no obstante incrementa la sensación de objetividad, oculta la relación que existe entre lo que el sujeto en formación conoce, en tanto sujeto implicado en su pregunta, y lo que descubre a partir de la construcción de un nuevo conocimiento acerca de la cultura escolar.

En contraste, en la etnografía retórica y narrativa el narrador en primera persona, implicado y portando su pasión y subjetividad, se inscribe en el texto que produce; preocupación que Gregory Bateson plantea en Naven (1958), al relacionar la producción de textos con la perspectiva subjetiva del autor. La autorreflexión y la implicación del investigador resultan en la actualidad, en contraste con las convenciones y pretensiones realistas, altamente significativas en los procesos de formación no sólo de los etnógrafos educativos, sino de los profesores que buscan profesionalizarse a través de la etnografía. En la escritura etnográfica actual, las 
diferencias a veces tajantes entre los supuestos iniciales y los descubrimientos empíricos constituyen la evidencia más palpable de que un nuevo conocimiento ha sido construido. Los maestros en formación necesitan aprender a valorar, en consecuencia, el significado de las marcas subjetivas, del uso de la primera persona y de la inclusión de sus experiencias, testimonios y trayectorias personales en la definición de las preguntas, la objetivación de los descubrimientos y la producción de sus escritos etnográficos (Schieffelin, 1976).

A pesar de que algunos antropólogos contemporáneos plantean los problemas implicados en la traducción, el significado y la función del intérprete (Marcus y Cushman, 1982, 1991), sostienen que los etnógrafos hemos aprendido a presentar nuestro material como si diéramos cuenta del punto de vista nativo suponiendo, en consecuencia, que la realidad "habla". Estos autores afirman, en contraste, que el etnógrafo describe, a fin de cuentas, el modo en que él, desde su propio marco cultural y subjetivo, ha vivido y leído la cultura de los otros. Si queremos caminar por el sendero de la implicación subjetiva necesitamos comprender que los escritos y los reportes etnográficos se elaboran a partir de tramas artificiosas, montadas y atravesadas por los sucesos enigmáticos y los marcos interpretativos que el autor desea subrayar intencionalmente.

En el taller que refiero concluimos que, para transitar por el sendero de la implicación subjetiva, necesitábamos incursionar por nuevos géneros narrativos. Reconocimos el trabajo pionero que realizan al respecto Pamela Rossi (1997) y Melanie Uttech (2001) en Estados Unidos, vía la utilización de la ópera, la poesía y la inteligencia creativa en los salones de clase multilingües y multiculturales; Carlos Miñana (1999) en Colombia, con directores de escuelas que escriben acerca de sí mismos y producen una escritura que se convierte en su lengua franca, en un código de interacción y en una escritura a muchas manos; y Nietta Lindenberg (1996) a partir del método basado en lo que define como una propuesta de autoría, impulsado con los maestros indígenas agroforestales de la Amazonía brasileña. Los miembros del taller reconocimos, en pocas palabras, que para objetivar subjetividades resultaba importante saber que los objetos se sujetan, mientras los sujetos se objetan.

\subsection{Implicación subjetiva y quiebras del borizonte subjetivo del intérprete}

Desde una postura congruente con las pretensiones realistas, solicitamos a los etnógrafos educativos en formación relegar la narración de su experiencia de campo a los prefacios, las notas a pie de página y los apéndices de sus informes, con alguna mención ocasional al respecto en los capítulos y la introducción. Enseñamos a nuestros alumnos que la experiencia subjetiva en campo ocupa un lugar marginal y privado, que impide su integración al informe escrito.

En contraste, para formarse como profesional del preguntar etnográfico, el profesor requiere apropiarse de una actitud abierta a la ambigüedad y a la naturaleza siempre cambiante, mas no contingente, de una realidad configurada a partir de la 
trama de significados intersubjetivos construidos en las comunidades, en las escuelas y en los salones de clases, en un momento histórico determinado. En este sendero de la formación, la experiencia en campo no cumple únicamente la función de responder a los cánones positivistas de la ciencia, ni se realiza con el fin de ratificar la subjetividad del intérprete. La experiencia en campo permite al profesional en formación, como sujeto implicado en su pregunta, reconfigurar sus horizontes significativos y representaciones educativas; proceso que se produce al fusionar: (i) la subjetividad del intérprete, (ii) la subjetividad de los sujetos interpretados y (iii) la subjetividad inherente a los modelos y conceptos teóricos producidos por otros autores (Bertely, 2000).

La etnográfica retórica y narrativa supone que esta experiencia interviene de modo significativo en la interpretación y, sobre todo, en las quiebras del horizonte subjetivo del intérprete, tal como lo define Michael Agar en su artículo Hacia un lenguaje etnográfico:

Una quiebra es una falta de concordancia entre el encuentro de uno con una tradición y las expectativas contenidas en los esquemas mediante los cuales uno organiza la propia experiencia. Uno modifica entonces los esquemas, o construye otros nuevos [...] hasta que la quiebra no es más un problema (Agar, 1982, 1991, 124).

Marcus y Cushman afirman, al respecto que:

Al detallar su propio shock o sorpresa epistemológica en puntos críticos de la narración, el etnógrafo conduce a sus lectores hacia una postura particular en relación con las diferencias culturales. [...] ofrece una reseña de su experiencia intelectual y de trabajo de campo con la que los lectores pueden identificarse, y mediante la autorreflexión del escritor como vehículo narrativo, deslizarse en una receptividad hacia descripciones que de otra manera podrian parecerles implausibles (Marcus y Cushman, 1982, 1991, 194).

Asunto al que alude también el mismo Agar cuando habla de la resolución de la quiebra:

[...] llamaremos proceso de resolución al proceso de trasladarse desde la quiebra hasta la comprensión [...]. Parafraseando a Gadamer, uno formula inicialmente un significado para la quiebra, pero luego lo desarrolla y lo refina en interacción con la "Cosa misma" (Agar, 1982, 1991, 123-124).

La resolución de la quiebra tiene lugar cuando los horizontes subjetivos se funden, la fusión de horizontes subjetivos es la hazaña del lenguaje, y ambas conllevan a una universalidad más elevada que plantea cambios en las representaciones escolares tanto del intérprete, como del informante (Rabinow, 1975; Blanchard, 1977). Considerando que para el intérprete las quiebras y las fusiones resultan más significativas y permanentes, en tanto los hallazgos y los descubrimientos le pertenecen, me resulta difícil imaginar una profunda transformación en las representaciones y las prácticas educativas sin intervención de la investigación en el proceso. 


\section{EL SENDERO FORMATIVO: COMPETENCIAS ETNOGRÁFICAS}

Para transitar de modo exitoso de la implicación subjetiva a la interpretación etnográfica en las comunidades, las escuelas y los salones de clases, resulta imprescindible contar con determinadas competencias indagatorias, sobre todo, cuando en el campo de la teoría curricular muchos autores discuten las opciones, los límites y las posibilidades de relacionar la docencia con la investigación, conformándose con demostrar la capacidad con que cuentan los profesores para construir estrategias que cuestionan y desbordan los límites y las determinaciones impuestos por los sistemas y las políticas estructurales y curriculares en materia educativa.

De este modo, a diferencia de las posturas que definen el currículum escolar como un plan que debe traducirse en prácticas de enseñanza específicas (Tyler, 1973; Taba, 1974; Stenhouse, 1984), existen otros enfoques que demuestran la existencia de una práctica educativa que no se ajusta al currículum prescrito y depende de múltiples factores ${ }^{2}$ (Sacristán, 1988). Factores entre los que se mencionan la selección de contenidos, las condiciones institucionales y las concepciones curriculares de los profesores (López Miranda, 2000), al igual que las posturas ideológicas que intervienen en la construcción de proyectos escolares específicos (Glazman e Ibarrola, 1983). La distinción entre plan de estudios y realidad curricular (Ibarrola, 1983), donde la realidad se independiza del proyecto a través de espacios y estructuras curriculares que intervienen en la definición local de los tiempos, los contenidos, las relaciones didácticas y la utilización de recursos distintos a los planeados, ha sido también documentada a partir de las diferencias que existen entre el currículum pensado y formal, por una parte, y el currículum vivido y real, por otra (Furlán, s.f.; Fernández, 1992).

Los aportes anteriores, sumados a derivados de la investigación etnográfica en educación y sus hallazgos acerca de las dimensiones que intervienen en el trabajo docente y en la configuración de la cultura escolar (Rockwell y Ezpeleta, 1983; Rueda, 1992; Bertely, 2000) resultan significativos, sobre todo, cuando de comprensión, diagnóstico y diseño curricular se trata. Desde estas perspectivas se asume que los profesores actúan en sus salones de clases contando con un margen de autonomía significativo (Rueda y otros, 1992; Díaz Barriga, 1995), seleccionan los contenidos y las modalidades didácticas de acuerdo con su historia personal, laboral y escolar (Aguilar, 1985) y construyen diversos modelos de docencia (Esquivel y Chehaibar, 1987).

No se resuelve del todo, sin embargo, el lugar que puede llegar a ocupar la investigación etnográfica en la formación de los maestros, dudándose en muchos casos acerca de esta posibilidad. Más aún, debido a la relación que se establece entre

2. Gimeno Sacristán (1988) señala ocho ámbitos que inciden en la práctica del currículum: político-administrativo, participación y control, ordenación del sistema educativo, producción de medios, creación cultural y científica, técnico-pedagógico, innovación y práctico-pedagógico, en López Miranda, 2000 . 
el concepto de vida cotidiana (Heller, 1977) y el proceso formativo experimentado por los profesores "lo cual ha sido mostrado con suficiencia en diversas etnografias escolares", se sostiene que los maestros aprenden a ser maestros en la práctica, pues gracias a ella se apropian de relaciones interpersonales y usos sociales, reproducen y transforman los marcos institucionales, y seleccionan "de manera inmediata y espontánea" modos alternativos de gestión educativa y de enseñanza.

Sin negar el valor de estos hallazgos en la producción de conocimiento nuevo acerca de los maestros y las escuelas, donde la investigación educativa aparece como un oficio diferente a la docencia (Achilli, 2000), lo que aquí me interesa mostrar es cómo el preguntar etnográfico puede incidir de modo efectivo en la formación de buenos profesores y, sobre todo, en la profunda transformación de las representaciones y las prácticas educativas. Considero que aún cuando algunos autores plantean estrategias de formación que buscan promover el conocimiento disciplinario y el trabajo académico y sistemático de los docentes (Díaz Barriga, 1988; Castillo y Chehaibar, 1991), y otros muestran el valor de la reflexión y el análisis de las concepciones y las prácticas de los maestros en su proceso de formación (López Miranda, 2000), no han sido explorados a profundidad y con suficiencia los senderos donde se relacionan la investigación etnográfica y la formación docente.

De este modo, a pesar de que existen alternativas probadas que colocan al conocimiento como el lugar donde se relacionan la investigación y la docencia (Achilli, 2000), deseo mostrar el valor que tiene la apropiación de determinadas competencias etnográficas en la formación de los profesores, sobre todo, cuando aprender a ser maestro en la práctica no implica, necesariamente, la capacidad de interpretar, sistematizar, formalizar y transformar la docencia en el sentido de generar una teoría educativa arraigada, sistemática y propia "producto de un proceso de apropiación", que pueda difundirse al interior de redes profesionales específicas.

La tesis que inspira este sendero estriba en que no obstante el conocimiento producido por los etnógrafos educativos puede intervenir en la definición de las políticas educativas, en la organización social de las escuelas y en el diseño de nuevos planteamientos curriculares, además de afectar el ambiente cotidiano en que se desenvuelven los profesores, dicha intervención e impacto resultan relativos porque los docentes traducen las reformas y los descubrimientos a partir de los marcos interpretativos y subjetivos que se han ido conformando en sus trayectorias personales.

Sin poner en duda otras alternativas de profesionalización que podrían garantizar la transformación de los marcos de interpretación de los maestros, considero que una experiencia que realmente forme y transforme las prácticas y las representaciones docentes "y en consecuencia las que se tejen y dan vida a las escuelas" depende del lugar que ocupa en dicha experiencia investigar y apropiarse de la profesión del preguntar etnográfico. Desde esta hipótesis, los profesores serán capaces de reconfigurarse, y no sólo de reproducir las nuevas orientaciones y las reformas curriculares en sentido instrumental o espontáneo, sólo y cuando puedan 
establecer un vínculo significativo, orgánico e intencional entre su labor docente y el preguntar en educación.

En otras palabras, considero que la investigación etnográfica no es capaz de modificar por sí sola las culturas escolares que estudia, y menos aún a los sujetos que participan en su construcción, a condición de suponer que el conocimiento puede ser transmitido de modo bancario. El sujeto que se transforma al estudiar las diversas realidades curriculares y culturas escolares "siendo fieles a la tradición constructivista" es, precisamente, el que pregunta, el que etnografía y el que pone a prueba sus experiencias y certezas previas, a fin de construir nuevos conocimientos y esquemas de interpretación. En este sendero resulta imposible separar la formación docente de la formación en investigación etnográfica, no en el sentido de introducirla de modo mecánico y precipitado, sino propiciando de modo progresivo, a lo largo del proceso formativo, la apropiación de algunas competencias relacionadas con el quehacer etnográfico en educación.

La efectiva relación entre formación docente y etnografía se logra cuando en el proceso formativo se promueve el dominio de las competencias que califican a los maestros para desempeñarse como verdaderos profesionales del preguntar en educación. Considero que estas competencias, inspiradas en la propuesta de Sonia Lavin (1990), son de dos tipos:

- las competencias básicas instrumentales relativas a dominio de las herramientas comunicativas propias de la profesión, integradas en las competencias lectora, escrita, oral y de razonamiento que garantizan el diálogo, la crítica y la comprensión intersubjetiva e intercultural, y

- las competencias contextuales relacionadas al desempeño de los profesores en los dominios teóricos y epistemológicos de la práctica profesional, incluidas las competencias para realizar un trabajo socialmente productivo, construir ambientes educativos pertinentes y adecuados, apropiarse de los conceptos y los métodos de las ciencias sociales y de las ciencias de la educación, así como lograr la convivencia democrática y la organización social y política en las escuelas.

Encuentro que las competencias instrumentales y contextuales a las que se refiere Lavin (1990), aplicadas en su caso a la escolarización en sentido amplio, se relacionan con la profesionalización docente y los dominios propios del quehacer etnográfico en educación. A continuación, menciono las competencias que requerirían los profesores para transformar sus representaciones y prácticas educativas de modo permanente. Me refiero a las competencias que requieren para realizar investigaciones de corte etnográfico o cualitativo en comunidades, escuelas y salones de clases, sin negar el valor de otras propuestas viables y útiles (Dalongeville y Huber, 2000; Elena Achilli, 2000). 


\subsection{Competencia lectora: ¿cómo leer textos y autores?}

Los profesores en formación suelen aproximarse a los libros de manera mecánica, pensando que las ideas, los conceptos y las propuestas que contienen constituyen conocimientos verdaderos e incuestionables, factibles de reproducirse sin mediación alguna en la gestión escolar y la práctica educativa. En contraste, los profesionales del preguntar etnográfico necesitan aprender que los textos son constructos de realidad y no la realidad en sí, producidos por autores en un momento y contexto cultural e histórico específico, donde la validez de los argumentos y los descubrimientos es relativa y corresponde a los debates propios de una época. Los textos y autores, en consecuencia, representan órdenes posibles de realidad que requieren ponerse en duda, ser leídos críticamente y asumirse como francas provocaciones a partir de las experiencias y las preguntas actuales de los lectores.

Contar con competencia lectora supone aprender a leer a otros «desde un lugar", lo cual representa una de las experiencias comunicativas más importantes en la profesión del preguntar etnográfico. En el acto de interpretar y leer un texto, y no sólo de repetir las ideas que en él se encuentran, se funden los horizontes significativos del autor y el lector (Heckman, 1984), por lo que aprender a leer un libro representa una de las primeras competencias etnográficas de carácter instrumental y contextual. No resulta exagerado afirmar que gracias a la competencia lectora también es posible descubrir la otredad, pues una buena reseña bibliográfica es aquella que considera las condiciones históricas, políticas y culturales en que se produce un texto, además de distinguir el horizonte significativo del autor de aquel desde el cual se interpreta lo leído.

Además, contar con competencia lectora supone comunicar a quienes comparten horizontes similares, vía oral y escrita, lo leído e interpretado; saber argumental que depende tanto del poder de convencimiento, la coherencia y la claridad verbal de lo dicho, como de la producción de escritos organizados, coherentes y claros acerca de los textos producidos por otros. En consecuencia, resulta fundamental leer y re-leer, escuchar y re-escuchar, escribir y re-escribir, interpretar y reinterpretar, porque contar con competencia lectora se traduce en la configuración de un pensamiento sistemático que integra las capacidades oral, escrita, de síntesis, crítica y razonamiento.

Si los profesores en formación aprenden a leer textos serán más capaces de leer los contextos socioculturales, institucionales y áulicos en que se desenvolverán más tarde, así como de promover una práctica docente fundada en el diálogo con los otros y en la constante modificación de sus representaciones y horizontes significativos. 


\subsection{Competencia teórica y metodológica: ¿cómo generar conceptos acerca de la realidad?}

Todo profesor se pregunta en algún momento de su trayectoria formativa: ¿qué es un marco teórico?, ¿cómo se construyen y operan empíricamente los conceptos y las categorías teóricas?, ¿cuáles son las actuales corrientes en ciencias sociales?, ¿qué se entiende por ciencias de la educación?, y ¿cuáles son los principales debates teóricos en el ámbito educativo? En congruencia con la definición de texto y autor, la teoría también aparece como un constructo de la realidad, de carácter abstracto, configurado por conceptos lógicamente ordenados, situados al interior de los modelos analíticos "nunca idénticos a la realidad vivida y experimentada cotidianamente" que caracterizan las distintas corrientes de pensamiento.

Contar con competencia teórica y metodológica implica comprender, caracterizar y situar por ejemplo, en el campo de las ciencias sociales, las diferencias y las coincidencias que se dan entre los modelos analíticos y los conceptos de las corrientes funcionalista, estructural funcionalista y estructuralista; las que existen entre los enfoques conductista, psicoanalítico, psicogenético y sociogenético en las ciencias de la educación; y las que se expresan en los debates entre las teorías de la reproducción y la resistencia en sociología de la educación.

Los profesores en formación necesitan saber que los modelos analíticos y los conceptos teóricos se construyen gracias a los ordenamientos categoriales, las clasificaciones de realidad y las denominaciones que, transitando de un nivel empírico a un nivel abstracto, construyen los propios investigadores "incluidos los etnógrafos" al interior de campos de conocimiento específicos. Percatarse del cómo o del modo en que se construye una teoría implica, en consecuencia, no sólo comprender lo que escribe un autor, sino leer metodológicamente los textos construidos.

Si bien un primer ejercicio deductivo, el cual implica probar la validez empírica de los conceptos teóricos construidos por otros, tiene implicaciones formativas invaluables en los futuros profesionales de la educación, propiciar la realización de lecturas metodológicas resulta muy importante para quien pretende formarse como profesional del preguntar etnográfico. Una lectura metodológica se define como la deconstrucción imaginaria de la trayectoria seguida por un etnógrafo educativo, como autor del texto, en relación al cómo y desde dónde:

- planteó la pregunta de investigación,

- delimitó el referente empírico,

- seleccionó las técnicas y los instrumentos,

- categorizó los hallazgos,

- trianguló empíricamente,

- trianguló teóricamente y

- presentó sus hallazgos.

En el libro Conociendo Nuestras Escuelas (Bertely, 2000) y en la introducción que hice al texto de Arcelia López Miranda (2000) aludo al debate en que Reba N.

(c) Ediciones Universidad de Salamanca

Teor. educ. 13, 2001, pp. 137-160 
Page e Yvonna S. Lincoln expusieron algunos retos implicados en la formación de investigadores educativos y, en particular, de etnógrafos. Afirmo en dicha introducción que:

Page, en un polo del debate, afirma que los etnógrafos educativos se forman, más que por hacer etnografías, por la lectura de etnografías que les permiten responder a cuestiones metodológicas del tipo: ¿por qué y para qué los etnógrafos se dedican a reunir las palabras de la gente?, ¿cómo otorgan significados específicos a los actos humanos? y, ¿en qué sustentan sus interpretaciones y evidencias? Al leer etnografías, diría Page, no sólo conocemos más acerca del modo en que se configura la realidad que nos interesa estudiar; además, nos percatamos del vínculo físico y emocional que se establece entre el etnógrafo y sus informantes; del grado en que la observación participante implica intimidad y un profundo sentido ético; y de la manera en que el investigador construye sus categorías y garantiza la validez de sus afirmaciones (López Miranda, 2000, 9).

Estoy convencida de que aunque la lectura de etnografías, definida como herramienta metodológica, apoya a los profesores en la identificación de modos alternativos de construir modelos analíticos, conceptos y teorías educativas arraigadas, requieren de competencias adicionales.

\subsection{Competencia epistemológica: ¿núcleo de sentido del trabajo educativo?}

Aunque mi propuesta de aprender a leer metodológicamente a los autores coincide en parte con el planteamiento de Page, en las obras mencionadas sostengo también que, en el polo contrario:

Yvonna Lincoln afirma que aunque la postura de Page resulta seductora, los etnógrafos en formación conocen muy poco acerca de los modelos de conocimiento que sustentan no sólo las investigaciones cualitativas, sino también las cuantitativas, lo que hace imprescindible introducirlos al manejo de las perspectivas epistemológicas que pueden ubicarlos en el contexto académico en que se producen los debates y se inserta su investigación (López Miranda, 2000, 9).

Las competencias epistemológicas a las que alude Yvonna Lincoln resultan también imprescindibles, sobre todo, si se considera que el tratamiento de este tipo de asuntos y más aún la palabra epistemología provocan serias resistencias entre los profesores.

Las perspectivas epistemológicas acerca del conocimiento y la realidad constituyen, no obstante las resistencias que provocan, el núcleo de sentido no sólo de la investigación en general, ni de la investigación educativa en particular, sino de la práctica educativa, resultando absurdo formar docentes cuya identidad y sentido profesional es ajeno a ellas. Sólo en el contexto de la neutralización del conocimiento en las escuelas, mencionado por Elena Achilli (2000), puede explicarse el temor que genera el tratamiento de los asuntos epistemológicos entre los maestros. Los 
cuestionamientos ¿qué implica conocer?, ¿qué es la realidad?, ¿cómo se conoce?, ¿qué es lo verdadero y lo falso?, ¿la realidad es objetiva o subjetiva?, apuntan al tratamiento de las competencias epistemológicas requeridas para relacionar la formación docente con la profesión del preguntar etnográfico.

Optar por determinadas teorías o corrientes de pensamiento, y no por otras, implica que el docente asume una posición espistemológica «en contraste con otras" en torno a qué es el conocimiento y la realidad. En ciencias sociales, lograr coherencia epistemológica supone optar entre posiciones objetivistas o subjetivistas, materialistas o idealistas, relativistas o deterministas, positivistas o hermenéuticas, holísticas o reduccionistas. Contar con competencia epistemológica implica, por ejemplo, optar entre interpretar realidades subjetivas "como lo haría un etnógrafo inspirado en la tradición hermenéutica, simbólica o interpretativa" o explicar lo real objetivo como algo que existe ahí, más allá del intérprete "como realidad definida de antemano, al igual que se comporta un etnógrafo positivista". Las opciones epistémicas pueden traducirse en perspectivas metodológicas parecidas en cuanto a la forma, pero distintas en su contenido e intención sustantivas.

Es gracias a este tipo de opciones que el profesor interesado en la profesión del preguntar etnográfico distingue entre metodologías cuantitativas "fundadas en el uso de estadisticas y encuestas" y cualitativas "apuntaladas, entre otras fuentes, en observaciones y entrevistas etnográficas, historias y trayectorias de vida, testimonios personales y textos narrativos". Distinción que le permitirá, en caso de optar por una metodología de corte cualitativo, saber argumentar por qué resulta significativo inscribir las tramas intersubjetivas escolares, dialogar con los otros, fusionar las perspectivas de los actores con la propia, y modificar los marcos epistemológicos de ambos horizontes de significado. Como mencioné, los debates epistemológicos propios del quehacer indagatorio se dan también en el campo pedagógico, en el sentido de reconocer al sujeto que aprende, en su particularidad, como reacción al modelamiento conductual que ha prevalecido, y aún prevalece, en nuestras escuelas.

\section{EL SENDERO INTERPRETATIVO: HACIA LA CONSTRUCCIÓN DE TEORÍAS EDUCATIVAS ARRAIGADAS}

Cuando el profesional del preguntar etnográfico se percata del modo en que está implicado subjetivamente en su pregunta, además de contar con las competencias mencionadas, está formado para:

- interpretar la realidad educativa,

- modificar las representaciones y las prácticas educativas, y

- construir teorías educativas emergentes y arraigadas (Glasser y Stauss, 1967).

En este punto del recorrido tenemos la certeza de poder relacionar la investigación etnográfica con la profesionalización docente. Interpretar la cultura escolar 
supone la capacidad de fusionar los horizontes subjetivos del etnógrafo y los distintos actores escolares: "autoridades, directores, maestros, alumnos y familias, entre otros". Los profesionales del preguntar educativo "léase buenos profesores", al documentar las representaciones y las prácticas escolares desde un lugar claramente definido, lo cual supone que son capaces de reconocer las perspectivas personales, teóricas y epistemológicas desde las cuales interpretan, comenzarán a poner a prueba sus juicios previos y a modificarse, haciendo de la quiebra, la autocrítica y la autocomprensión una práctica habitual. Imagino que, en este horizonte profesional, surgirán entre los profesores preguntas del tipo: ¿cómo pude suponer que la realidad educativa era así cuando existen otras representaciones, prácticas y expectativas en torno a ella?

Concatenada a la anterior, la pregunta ¿podría pensar y hacer lo que hago de modo distinto?, encierra en su aparente ingenuidad la posibilidad de generar teorías educativas arraigadas, definidas como los constructos analíticos y conceptuales producidos por los propios maestros, en materia de gestión institucional o en el ámbito pedagógico, en contextos comunitarios, escolares y áulicos específicos; generación teórica que exige de los profesionales no sólo de imaginación y creatividad social (Uttech, 2001), sino de capacidades específicas para funcionar como verdaderos estrategas docentes.

La capacidad estratégica entre los docentes no sólo supone que construyen estilos institucionales y de enseñanza adecuados, espontáneos y cotidianos (Hargreaves, 1985), sino teorías educativas emergentes ancladas en los principios de equidad y pertinencia educativas. La equidad, definida como el derecho que tienen todos los alumnos de acceder a los contenidos y las competencias nacionales y universales, sitúa al estratega docente en la sociedad amplia y las demandas ciudadanas. La pertinencia educativa, mientras tanto, exige al docente saber cómo adaptar, traducir y generar apropiaciones escolares a nivel local, relacionadas con las expectativas y condiciones de vida de los usuarios. En consecuencia, considero que la calidad educativa en nuestros países sólo será posible cuando los profesores de grupo sean capaces de:

- observar, escuchar y aprender de otros inscribiendo e interpretando las categorías sociales que se expresan en las representaciones y en las prácticas que configuran una cultura escolar específica;

- asignar nuevos sentidos a las representaciones y a las prácticas escolares mediante el descubrimiento de las relaciones emergentes, significativas y laterales a las hegemónicas, enunciadas en conceptos y teorías propias; y

- descubrir que los conceptos y las teorías propias pueden dialogar con los conceptos y las teorías educativas legadas por otros.

En términos metodológicos, el triángulo invertido al que aludo en Conociendo Nuestras Escuelas (Bertely, 2000), expresión metafórica del accionar interpretativo en etnografía, podría funcionar como un modelo formativo para los 
nuevos profesionales en educación. Para poder moverse en dicho triángulo los profesores necesitan saber:

- cómo documentar lo que piensan, dicen y hacen los actores educativos en las escuelas y salones de clases (categorías sociales),

- desde dónde y cómo organizar y conceptualizar lo que piensan, dicen y hacen los actores (categorías del intérprete) y

- cómo han analizado realidades similares otros autores (categorías teóricas).

Sólo entonces los maestros en formación y en servicio, y no sólo los etnógrafos educativos, serán capaces de producir teorías educativas arraigadas que les resulten significativas. El horizonte de sentido de este tercer sendero, en conclusión, vislumbra la transformación de las representaciones y las prácticas educativas desde los actores y, en consecuencia, la invención y configuración de viejos, actuales y futuros imaginarios y tradiciones escolares capaces de vitalizar, desde dentro, identidades y comunidades escolares en crisis (Galindo, 1993; Hobsbawm y Ranger, 1983; Anderson, 1993).

\section{EL SENDERO DEMOCRÁTICO: COMUNIDADES DE INDAGACIÓN INCLUYENTES}

Por último deseo aclarar que las tesis expuestas son relativas en tanto se configuran a partir de mi propia subjetividad, historia de vida y trayectoria profesional. No es lo mismo reflexionar acerca del modo de relacionar la etnografía con la formación de mejores enseñantes en y para nuestras escuelas, que pretender asignar un estatuto de validez absoluto a mis afirmaciones y propuestas.

En otro artículo (Bertely, 1999) cuestionó los mecanismos de exclusión y selectividad que reproduce la comunidad académica al descalificar, a partir de los paradigmas científicos con los que comulgan, a los diversos agentes sociales individuos, instituciones y organizaciones" que, en contextos distintos al académico, producen también conocimiento en y para las escuelas.

Para reconocer nuestros límites requerimos acceder a una definición de validez múltiple, relacionada con las diversas condiciones de producción, difusión e impacto del conocimiento generado. La validez es múltiple y relativa porque los agentes que intervienen en la construcción de la cultura escolar producen conocimientos que resultan socialmente funcionales, dependiendo del ámbito de incidencia en que se desenvuelven (Hekman, 1984). Esta relatividad no implica aislamiento porque en la negociación política y el consenso social se construyen tanto la legitimidad siempre precaria de los hallazgos y proyectos generados, como el conjunto de representaciones y prácticas comunes y distintivas que se producen en torno a un campo de conocimiento específico. En consecuencia, lo que la comunidad de etnógrafos educativos define como válido y adecuado lo es, sobre todo, para los miembros que participamos en esta comunidad, sin que ello implique ignorar que 
dependemos de un consenso que se teje más allá de nuestras fronteras disciplinarias, así como la validez de otras perspectivas con las que deberíamos dialogar.

En base a determinados estudios, diagnósticos, seguimientos y evaluaciones, los legisladores, los consultores y los funcionarios de organismos nacionales e internacionales participan en la definición de políticas educativas de amplio alcance. Las organizaciones no gubernamentales irrumpen como los nuevos agentes de negociación entre la sociedad política y la sociedad civil, mientras los equipos técnicos definen los objetivos, los contenidos y las metodologías de los planes y los programas de estudio oficiales. Agentes que producen conocimientos socialmente funcionales, relacionados con la negociación política, la toma de decisiones, la gestión educativa y el diseño curricular, pero situados en la exterioridad del campo académico.

En las instituciones formadoras de docentes, los formadores, los futuros profesores y los maestros en ejercicio, incorporan algunos de los conocimientos producidos por los investigadores a los proyectos de tesis, enfrentan el reto de traducir las políticas y reformas curriculares en espacios concretos y, considerados como una especie de aprendices permanentes, no suelen compartir sus hallazgos y propuestas con los académicos. Al igual que estos agentes, quienes ocupan puestos relacionados con la gestión educativa en los niveles de educación básica, media-superior y superior, o coordinan áreas de investigación, docencia, titulación, difusión y extensión académica, se preguntan en qué medida lo que hacen puede ser catalogado como nuevo conocimiento.

Los saberes educativos de los llamados usuarios cuentan aún con un estatuto de validez menor, porque las madres y los padres de familia, así como los alumnos y las alumnas, no participan en los eventos académicos. Sin negar el valor ético que se deriva de incorporar estas voces en los descubrimientos etnográficos, su utilización puede explicarse a partir cle un interés puramente académico: lograr que nuestros reportes de investigación y ponencias resulten verosímiles a nuestros pares.

La comunidad académica excluye a los usuarios a pesar de que son los agentes fundamentales que intervienen en el proceso de escolarización, asignan legitimidad de facto al conocimiento educativo producido, y garantizan el consenso de las políticas educativas implantadas. Los padres y madres de familia, sin embargo:

- saben más acerca de sus hijos que los propios maestros,

- imaginan formas alternativas de enseñanza para sus hijos,

- incorporan o rechazan las innovaciones curriculares,

- se debaten entre las perspectivas tradicionales y modernas en materia pedagógica y,

- esperan que las escuelas respondan a sus expectativas y condiciones de vida y trabajo.

La distancia entre el mundo académico y los nuevos liderazgos sociales resulta también sintomática. Las propuestas educativas generadas por la nueva intelectualidad indígena apenas comienzan a ser estudiadas por académicos que buscan cómo responder a las expectativas y particularidades lingüísticas, culturales y productivas 
de los pueblos étnicamente distintivos en México. Por su parte, las organizaciones no gubernamentales se desenvuelven al margen del campo académico porque comulgan con paradigmas inspirados en la investigación-acción participativa.

En todos los casos se trata de agentes sociales que producen saberes y conocimientos estratégicos, multideterminados y funcionales que, por su carácter asistemático y sometido a imponderables, se sitúan en la exterioridad del campo académico; exclusión que se da no obstante sus voces y prácticas constituyen la materia prima de nuestras indagaciones e inciden en el consenso social en torno al proceso de escolarización.

La presencia de nuevos agentes sociales en la producción del conocimiento educativo exige construir comunidades de indagación incluyentes que articulen lo común con lo distintivo y, en pocas palabras, estimulen el diálogo entre los distintos agentes sociales. Entre otros, corresponde a los enseñantes discutir y definir los sentidos, los usos y las prácticas que los lleven a configurar una nueva tradición en el campo de la investigación educativa, avanzar en la producción de conocimientos en su ámbito de influencia y construir sus propias comunidades de indagación.

Para superar la realidad virtual en que suele reproducirse la academia y acortar la brecha entre los investigadores educativos, la sociedad política y la sociedad civil, me parece imprescindible reintegrar a los profesores su capacidad no sólo de traducir los conocimientos generados por otros, sino de producir teorías educativas arraigadas. En este sentido, en materia de gestión educativa y práctica pedagógica, los profesionales del preguntar etnográfico pueden contribuir en la fusión de los horizontes significativos que intervienen en la configuración de la cultura escolar. Afirmé antes que la fusión de horizontes, como producto de la interpretación etnográfica, es la hazaña del lenguaje. Lo importante para los profesores es producir un lenguaje a muchas manos que, además de objetivarse en notas de campo y reportes de investigación, incida en la transformación de sus representaciones y prácticas educativas, así como en la construcción de espacios donde puedan socializar el modo en que en sus interpretaciones se fusiona su propia perspectiva con la de los académicos, los legisladores, los consultores, los funcionarios de organismos nacionales e internacionales, los formadores de maestros, los directivos, los padres de familia y los líderes comunitarios. Estaremos entonces preparados para transitar del profesor usuario del conocimiento educativo, al profesional productor de conocimientos apuntalados en el preguntar etnográfico.

Para abatir la inequidad y baja calidad de los servicios ofrecidos, las altas tasas de reprobación y deserción escolares, la dudosa pertinencia de los objetivos, contenidos y métodos, así como la crisis de nuestros modelos educativos, no basta con difundir los hallazgos derivados de la investigación educativa, ni encontrar a partir de ahí los caminos más adecuados para comprender y enfrentar los retos planteados (Ibarrola, 1987). Requerimos, además, de espacios plurales donde los diversos agentes que participan en la configuración de la cultura escolar comuniquen sus conocimientos, así como de una profunda transformación de las representaciones y las prácticas de los profesores. 


\section{BiBLIOGRAFÍA}

ACHILLI, E. L. (2000) Investigación y formación docente. Argentina, Laborde Editor.

AGUILAR, C. (1985) La definición cotidiana del trabajo de los maestros, en ROCKWELL, E. (comp.). Ser maestro, estudios sobre el trabajo docente. México, Secretaría de Educación Pública/Caballito.

ANDERSON, B. (1993) Comunidades imaginadas. México, Fondo de Cultura Económica.

BATESON, G. (1958) Naven. Stanford, Stanford University Press.

BERTELY, M. (1998) Infancia indígena y su derecho a una educación de calidad, Revista Huaxyácac, n 14. México, Instituto Estatal de Educación de Oaxaca.

- (1998a) Educación indígena del siglo XX en México, en LATAPÍ, P. (coord.). Un siglo de la educación en México. México, Biblioteca Mexicana del Siglo XXI, Fondo de Cultura Económica.

- (2000) Conociendo nuestras escuelas. Un acercamiento etnográfico a la cultura escolar. México, Buenos Aires Barcelona, Colección Maestros y Enseñanza, Editorial Paidós.

- (inédito) La investigación educativa en el Estado de México: reflexiones en torno a la construcción institucional y social de un campo profesional. Chalco, Estado de México, Instituto Superior de Ciencias de la Educación del Estado de México.

- (en prensa) La escritura etnográfica: ¿desde dónde y para quién?, en Taller: IX Simposio Interamericano de Investigación Etnográfica en Educación. México, Universidad Pedagógica Nacional/Ajusco, 24-25 octubre, 2000.

BERTELY, M. y CORENSTEIN, M. (1994) Panorama de la investigación etnográfica en México: una mirada a la problemática educativa, en RUEDA, M.; DELGADO, G. y JACOBO, Z. La etnografía en educación: panorama, prácticas y problemas. México, Centro de Investigaciones y Servicios Educativos. Universidad Nacional Autónoma de México.

BLANCHARD, K. (1977) The expanded responsabilities of long term informant relationship, Human Organization, 36, 66-69.

GRIGGS, J. L. (1970) Never un Anger: portrait of an eskimo family. Cambridge, Harvard University Press.

CASTILLO, R. y CHEHAIBAR, L. (1991) Etnografỉa y formación docente, en RUEDA, M.; DELGADO, G. y CAMPOS, M. A. (comps.). El aula universitaria. Aproximaciones metodológicas. México, Centro de Investigaciones y Servicios Educativos, Universidad Nacional Autónoma de México.

COMEXANI (1998) Los bechos se burlan de los derechos. IV Informe sobre los derechos y la situación de la infancia en México 1994-1997.

DALONGEVILLE, A. y HUBER, M. (2000) (Se) Former par les situations-problèmes. Des déstabilisations constructives. Lyon, Chronique Sociale, Pédagogie-Formation.

DEVERAUX, G. (1977) De la ansiedad al método en las ciencias del comportamiento. México, Siglo XXI.

DE IBARROLA, M. y otros (1983) Docencia (documento de trabajo). México, Guadalajara, Jal., Comisión Estatal para la Integración del Sistema Formador y Actualizador de Docentes.

DE IBARROLA, M. (1987) La formación del investigador en México, Avance y Perspectiva, $\mathrm{n}^{\circ}$ 33. México, Centro de Investigaciones y Estudios Avanzados, Instituto Politécnico Nacional.

DÍAZ BARRIGA, A. (1988) Evolución de la formación pedagógica de los profesores universitarios. Conferencia: Foro Nacional sobre Formación de Profesores. México, UNAM/SEP/ANUIES. 


\section{- (1995) Tarea docente. México, Nueva Imagen.}

ESQUIVEL, J. E. y CHEHAIBAR, L. (1987) Profesionalización de la docencia en México. México, Centro de Estudios sobre la Universidad, Universidad Nacional Autónoma de México.

FERNÁNDEZ, H. H. (1992) Dimensiones del currículum. Subproducto del proyecto de investigación: Evaluación curricular de un programa de formación y actualización de docentes. México, Instituto Superior de Ciencias de la Educación del Estado de México.

FURLÁN, J. A. (s/f) El curriculum pensado y el curriculum vivido (mimeo). México, Escuela Nacional de Estudios Profesionales, Universidad Nacional Autónoma de México.

GALEANA, R. (1997) La infancia desertora. Fundación SNTE para la Cultura del Maestro Mexicano A.C.

GALINDO, J. (1993) Desde la cultura y más allá de la cultura. Notas sobre algunas reflexiones metodológicas, en LOPERA, E.; DÍEZ, H.; GALINDO, J. y otros. Investigación cualitativa. Confrontación y prospectiva. Medellín, Colombia, Centro de Investigaciones Educativas y Pedagógicas, Universidad de Antioquía.

GLASSER, B. y STRAUSS, A. (1967) The discovery of grounded theory. Chicago, Aldine.

GLAZMAN, R. y DE IBARROLA, M. (1983) Diseño de planes de estudio: modelo y realidad curricular. México, Departamento de Investigaciones Educativas, Cinvestav, IPN.

HARGREAVES, A. (1985) El significado de las estrategias docentes, en ROCKWELL, E. (comp.). Ser maestro, estudios sobre el trabajo docente. México, Secretaría de Educación Pública, Caballito.

HEKMAN, S. (1984) Action as a text: Gadamer's hermeneutics and the social scientific analysis of accion, Journal for Theory of Social Behavior, vol. 14, $\mathrm{n}^{\circ}$ 3, october.

HELLER, A. (1977) Sociología de la vida cotidiana. Barcelona, Península.

- (1993) Teoría de los sentimientos. México, Editorial Fontamara.

HOBSBAWM, E. y RANGER, T. (1983) The invention of tradition. Cambridge, Universit! Press.

LAVIN, S. (1990) Competencias básicas para la vida: intento de una delimitación conceptual (mimeo). México, Centro de Estudios Educativos, A.C.

LÓPEZ MIRANDA, M. A. (2000) Enseñar en Comala. Concepciones y prácticas de los maestros: un recurso para la formación docente. México, Universidad de Guadalajara.

OWUSU, M. (1978) Ethnography of Africa: the usefulness of the useless, American Antbropologist, 80, 310-334.

RABINOW, P. (1975) Symbolic domination: cultural form and historical change in Morocco. Chicago, University Chicago Press.

REYNOSO, C. (comp.) (1991) El surgimiento de la antropología posmoderna. México, Gedisa Editorial.

ROCKWELL, E. y EZPELETA, J. (1983) La escuela, relato de un proceso de construcción inconcluso. Departamento de Investigaciones Educativas, Cinvestav, IPN.

RODRÍGUEZ, P. G. (coord.) (1999) Delimitación y validez de la investigación educativa. México, Centro de Estudios Educativos, A.C.

ROSALDO, M. Z. (1980) Knowledge and passion: Ilongot notions of self and social life. Nueva York, Cambridge University Press.

- (1980a) Ilongot headbunting 1883-1974: a study in society and history. Stanford, Stanford University Press. 
RUEDA, M. (1992) La educación universitaria: la función de la clase, en RUEDA, M. y CAMPOS, M. A. Investigaciones etnográficas en educación. México, Centro de Investigaciones y Servicios Educativos, Universidad Nacional Autónoma de México.

RUEDA, M. y otros. (1992) Prácticas pedagógicas en una institución superior. Third Interamerican Symposium of Ethhography and Classroom. Nuevo México, USA.

SACRISTÁN, J. G. (1988) El curriculum, una reflexión sobre la práctica. Madrid, Morata.

SCHIEFFELIN, E. L. (1976) The sorrow of the lonely and the burning of the dancers. Nueva York, Saint Martin's.

STENHOUSE, L. (1984) Investigación y desarrollo del curriculum. Madrid, Morata.

TABA, H. (1974) Elaboración del currículo. Buenos Aires, Troquel.

TYLER, R. (1973) Principios básicos del currículum. Buenos Aires, Troquel.

UTTECH, M. (2001) Imaginar, facilitar, transformar. Una pedagogía para el salón multigrado y la escuela rural. México, Buenos Aires, Barcelona, Colección Maestros y Enseñanza, Editorial Paidós.

WEST, T. (coord.) (1995) Educación, cultura y procesos sociales. La investigación educativa en los ochenta. Perspectivas para los noventa. México, Consejo Méxicano de Investigación Educativa, Universidad Nacional Autónoma de México. 